\title{
Evaluation of Rice Husk Ash from Different Rice Varieties from Nigeria: Silica Content and Lethality to Two Storage Beetles
}

\author{
Atewoja Y.Y., Ofuya T.I., Idoko J.E., Adebayo R.A.
}

\begin{abstract}
This study was conducted under ambient laboratory conditions of the Research Laboratory, Department of Crop, Soil and Pest Management, The Federal University of Technology, Akure, Nigeria, to evaluate the lethality of rice husk ash (RHA) of six rice varieties from different geographical areas in Nigeria to the cowpea seed beetle, Callosobruchus maculatus Fabricius and the maize weevil, Sitophilus zeamais Motschulsky in relation to the silica content. RHA was produced and the silica content determined using standard methods. The lethality of the RHAs was tested at $0.0,0.2,0.4$, 0.6, 0.8 and $1.0 \mathrm{~g} / 20 \mathrm{~g}$ of grain dosages against 10 adult beetles. The silica content ranged from $54.65 \%$ to $90.45 \%$ being highest for Jemila variety RHA and lowest with Igbemo RHA. Irrespective of dosage and duration of exposure, all the RHAs generally produced significant mortality of $C$. maculatus and $S$. zeamais in comparison with the control. The Jemila variety RHA was most lethal to the two storage beetles followed by the FARO 44 and Kilaki RHAs. RHA lethality to the two beetles was significantly positively correlated with silica content. RHA with high silica content is suited for inclusion in insecticidal botanical cocktails and integrated stored grain protection.
\end{abstract}

Index Terms - Rice husk ash, silica content, Callosobruchus maculatus, Sitophilus zeamais, mortality.

\section{INTRODUCTION}

Many varieties of rice, Oryza sativa $\mathrm{L}$ are cultivated in different ecological zones of Nigeria; some of which are local and others, improved or hybrid types. A major by-product or waste product from rice milling is rice husk or hull. In majority of rice producing developing countries like Nigeria much of the husk produced from processing of rice is either burnt or dumped as waste and poses a great environmental threat [1]. Commercial use of rice husk and its ash would undoubtedly be an alternative solution to the disposal problem [2]. Ash obtained from burnt plant materials perhaps one of the commonest plant product that has been used to mitigate grain damage by insects in African traditional storage systems. Wood ash and ash from different plants species have been investigated and varying efficacies have been reported for different storage pest insects [3]-[8]. Ash obtained from rice husk appears to be particularly efficacious against storage beetle pests [9] [10] [2] [11]. The toxicity of

Atewoja Y.Y., Department of Crop, Soil and Pest Management, The Federal University of Technology, PMB 704, Akure, Nigeria.

Ofuya T.I., Department of Crop, Soil and Pest Management, The Federal University of Technology, PMB 704, Akure, Nigeria.

Idoko J.E., Department of Crop, Soil and Pest Management, The Federal University of Technology, PMB 704, Akure, Nigeria.

Adebayo R.A., Department of Crop, Soil and Pest Management, The Federal University of Technology, PMB 704, Akure, Nigeria. rice husk ash to insects has been principally attributed to its silica content [12]. In this paper we report the results of an investigation of the silica content of rice husk ash of rice varieties from different geographical rice growing areas of Nigeria. We further report results of studies on the lethality of these ashes to adults of the cowpea seed beetle, Callosobruchus maculatus Fabricius and the maize weevil, Sitophilus zeamais Motschulsky, the major storage pests of cowpeas and maize grain, respectively, in Nigeria.

\section{MATERIALS AND METHODS}

The ereaxperiment was conducted in the Research Laboratory Department of Crop, Soil and Pest Management, the Federal University of Technology, Akure, Nigeria, under prevailing laboratory conditions: temperature of $28 \pm 2^{\circ} \mathrm{C}$ and relative humidity of $75 \pm 5 \%$.

\section{A. Insect Culture, cowpea and maize grain}

Adults of Callosobruchus maculatus and Sitophilus zeamais were obtained from already infested cowpea and maize grains from Institute of Agricultural Research and Training (IAR\&T), Ibadan, Nigeria. C. maculatus and S. zeamais was reared on clean Ife Brown variety of cowpea and SUWAN -1 yellow variety of maize, respectively, in 51 plastic containers holding $500 \mathrm{~g}$ of grain. The cowpea and maize grain were obtained from IAR\&T. The insect cultures were recycled monthly by sieving out adults from damaged grain and introducing them into clean grain in other plastic containers under 12:12 light and dark regime. Before use, the cowpea and maize grain were disinfested in a deep freezer for 2 weeks and thereafter spread out on a laboratory bench for acclimation.

\section{B. Rice husk and ash}

The rice husks used for the research were obtained from different varieties and rice mills: Nupe rice from Lafiagi in Kwara State; Kilaki rice from Das in Bauchi State; Jemila rice from Kachia in Kaduna State; FARO 44 rice from Omor in Anambra State; Aroso rice from Ogoja in Cross Rivers State; and Igbemo rice from Igbemo in Ekiti State. The husks were purified by removing all extraneous objects. They were subsequently pre-ashed by setting on fire and allowed to burn completely. Thereafter, the RH charcoals were allowed to cool and transferred into a Muffle furnace to produce ash at temperature of $550^{\circ} \mathrm{C}$ [13]. Each ash was kept separately in a plastic container with firm cover and stored in the laboratory until when needed.

\section{Determination of silica content ash}

The silica content of each ash was determined following the TAPPI 244om-93 (1999) method. Ash obtained as previously described was digested with $5 \mathrm{ml}$ of $6 \mathrm{M} \mathrm{HCl}$ and evaporated 
on a stream bath to dryness. After evaporation, another $5 \mathrm{ml}$ of $6 \mathrm{M} \mathrm{HCl}$ was added and evaporated. The addition of another $5 \mathrm{ml}$ of $6 \mathrm{M} \mathrm{HCl}$ to the residue was followed by heating, and then the solution was diluted with $20 \mathrm{ml}$ of distilled water. Hot distilled water was used to wash the residue on ash free filter paper. After sufficient washing to remove chloride, the ash free filter paper along with residue was placed in the crucible and ignited at $525^{\circ} \mathrm{C}$ for $6 \mathrm{~h}$. After cooling, the silica weight (g) was taken. The experiment was done in triplicate. The following formula was used to obtain the silica content:

$\%$ Silica content $=\frac{\mathrm{c}}{\mathrm{d}} \mathrm{x} \frac{100}{1}$

Where;

$\mathrm{c}=$ weight of the silica $(\mathrm{g})$

$\mathrm{d}=$ weight of the sample $(\mathrm{g})$

D. Lethality of ash tests

Twenty grams of clean cowpea and maize seeds were weighed into $250 \mathrm{ml}$ plastic containers and treated with each ash type at $0.2,0.4,0.6,0.8$ and $1.0 \mathrm{~g}$, respectively, to which was added 10 adult beetles ( 5 males and 5 females, one-day old in case of $C$. maculatus, but unsexed for $S$. zeamais and less than 1 week old). There was an untreated control. Adult beetle mortality was observed at 24,48 , and $72 \mathrm{~h}$ for $C$. maculatus on cowpea, and at 24, 48, 72 and $96 \mathrm{~h}$ for $S$. zeamais on maize, post treatment. The experiment was set up in a completely randomized design and each treatment was replicated three times.

E. Data Analysis

Mortality data were arcsine transformed and subjected to one-way analysis of variance (ANOVA) consistent with completely randomized design. Means were separated using Tukey's Honestly Significant Difference Test. Mortality data was further correlated with silica content and also subjected to probit regression analysis to calculate the $\mathrm{LD}_{50}$ of the treatments [14].

\section{RESULTS}

Figure 1 shows silica content of RHA of rice varieties from different geographical areas in Nigeria. Jemila rice variety RHA (from Kaduna State) had the highest percentage silica content of $90.45 \%$ which was significantly $(p<0.05)$ different from others except FARO 44 rice variety RHA (from Anambra State) and Kilaki rice variety RHA (from Bauchi State), that recorded $86.58 \%$ and $85.97 \%$, respectively. Igbemo rice variety RHA (from Ekiti State) recorded the lowest silica content of $54.65 \%$.

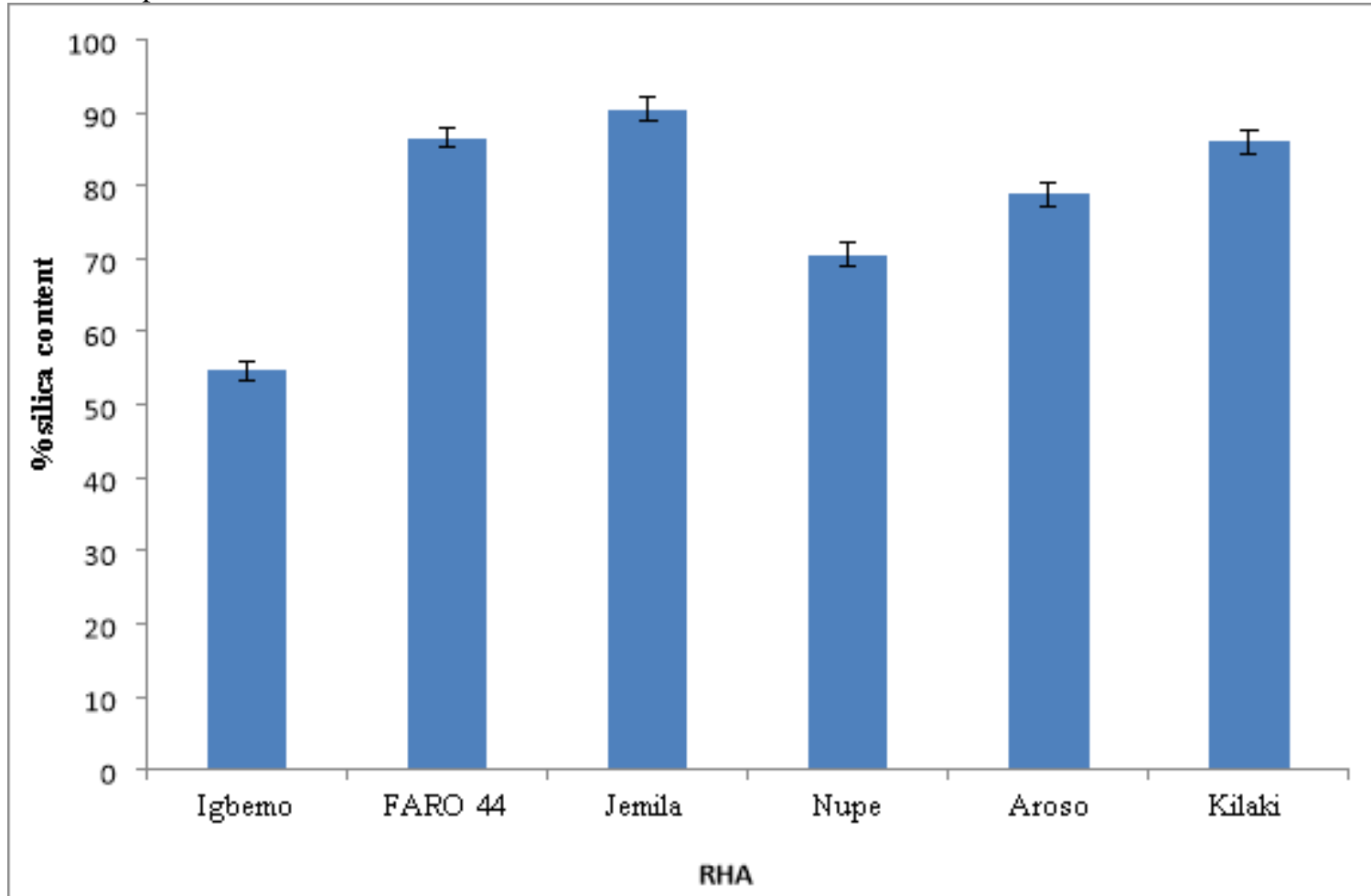

Fig.1. \% silica content of rice husk ash (RHA) produced from six different rice varieties grown in Nigeria.

Tables 1- 5 show the mortality of $C$. maculatus at various times in cowpea grain treated with RHAs from six different Nigerian rice varieties at dosages of $0.2-1.0 \mathrm{~g} / 20 \mathrm{~g}$ of grain in comparison with the control ( $0.0 \mathrm{~g}$ dosage $)$. Irrespective of dosage and duration of exposure, all the RHAs generally produced significant $(\mathrm{p}<0.05)$ mortality of $C$. maculatus in comparison with the control. Table 1 showed that at $0.2 \mathrm{~g}$ dosage, only the Jemila RHA produced above $40 \%$ mortality of the beetle within $24 \mathrm{~h}$ post treatment which was significantly $(\mathrm{p}<0.05)$ different from others except the FARO 44 RHA which produced $36.67 \%$ mortality. Within 72 h post treatment, all the ashes from different locations achieved $100 \%$ mortality of the insect at $0.2 \mathrm{~g}$ dosage except the Igbemo RHA that was unable to achieve up to $50 \%$ mortality of the insect (Table 1). Within $24 \mathrm{~h}$ post treatment, $0.4 \mathrm{~g}$ of RHA from Kaduna recorded $66.67 \%$ insect mortality and was 
significantly $(\mathrm{p}<0.05)$ different from others. At the same dosage at $72 \mathrm{~h}$ post treatment, all the RHAs produced $100 \%$ mortality except the Igbemo RHA (Table 2). All the RHAs at $0.6 \mathrm{~g}$ dosage achieved more than $50 \%$ mortality within $48 \mathrm{~h}$ of exposure except Nupe and Igbemo RHAs that were able to produce $43.33 \%$ and $46.67 \%$ mortality, respectively (Table 3 ). Table 4 showed that the Jemila RHA was significantly $(\mathrm{p}<0.05)$ different from the other RHAs achieving up to $80 \%$ beetle mortality within $24 \mathrm{~h}$ of exposure. At $72 \mathrm{~h}$ post treatment, all RHAs achieved $100 \%$ mortality of $C$. maculatus except Igbemo RHA that achieved $63.33 \%$ beetle

Table 1 . Mortality of $C$. maculatus exposed to $0.2 \mathrm{~g}$ dosage of RHA from six different rice varieties from Nigeria

\begin{tabular}{llcl}
\hline RHA & \% adult mortality in hours post treatment & \\
& $\mathbf{2 4}$ & $\mathbf{4 8}$ & $\mathbf{7 2}$ \\
\hline Nupe & $10.00 \pm 0.00^{\mathrm{a}}$ & $30.00 \pm 0.00^{\mathrm{b}}$ & $100.00 \pm 0.00^{\mathrm{c}}$ \\
Jemila & $46.67 \pm 3.33^{\mathrm{c}}$ & $70.00 \pm 0.00^{\mathrm{c}}$ & $100.00 \pm 0.00^{\mathrm{c}}$ \\
FARO 44 & $36.67 \pm 3.33^{\mathrm{bc}}$ & $73.33 \pm 6.67^{\mathrm{c}}$ & $100.00 \pm 0.00^{\mathrm{c}}$ \\
Kilaki & $33.33 \pm 3.33^{\mathrm{b}}$ & $66.67 \pm 6.67^{\mathrm{c}}$ & $100.00 \pm 0.00^{\mathrm{c}}$ \\
Aroso & $33.33 \pm 3.33^{\mathrm{b}}$ & $66.67 \pm 3.33^{\mathrm{c}}$ & $100.00 \pm 0.00^{\mathrm{c}}$ \\
Igbemo & $0.00 \pm 0.00$ & $20.00 \pm 0.00^{\mathrm{b}}$ & $46.67 \pm 3.33^{\mathrm{b}}$ \\
Control & $0.00 \pm 0.00$ & $0.00 \pm 0.00$ & $0.00 \pm 0.00$ \\
\hline
\end{tabular}

Each value is mean \pm standard error of three replicates. Values followed by the same alphabet are not significantly ( $p>0.05$ ) different from each other using Tukey's Test.

Table 2. Mortality of $C$. maculatus exposed to $0.4 \mathrm{~g}$ dosage of RHA from six different rice varieties from Nigeria

\begin{tabular}{llll}
\hline RHA & \multicolumn{3}{l}{ \% adult mortality in hours post treatment } \\
& $\mathbf{2 4}$ & $\mathbf{7 2}$ & \\
\hline Nupe & $16.67 \pm 3.33^{\mathrm{b}}$ & $40.00 \pm 5.77^{\mathrm{c}}$ & $100.00 \pm 0.00^{\mathrm{c}}$ \\
Jemila & $66.67 \pm 3.33^{\mathrm{d}}$ & $86.67 \pm 3.33^{\mathrm{d}}$ & $100.00 \pm 0.00^{\mathrm{c}}$ \\
FARO 44 & $46.67 \pm 3.33^{\mathrm{c}}$ & $80.00 \pm 0.00^{\mathrm{d}}$ & $100.00 \pm 0.00^{\mathrm{c}}$ \\
Kilaki & $43.33 \pm 3.33^{\mathrm{c}}$ & $80.00 \pm 5.77^{\mathrm{d}}$ & $100.00 \pm 0.00^{\mathrm{c}}$ \\
Aroso & $36.67 \pm 3.33^{\mathrm{c}}$ & $70.00 \pm 0.00^{\mathrm{d}}$ & $100.00 \pm 0.00^{\mathrm{c}}$ \\
Igbemo & $10.00 \pm 0.00^{\mathrm{ab}}$ & $26.67 \pm 3.33^{\mathrm{b}}$ & $50.00 \pm 5.77^{\mathrm{b}}$ \\
Control & $0.00 \pm 0.00^{\mathrm{a}}$ & $0.00 \pm 0.00^{\mathrm{a}}$ & $0.00 \pm 0.00^{\mathrm{a}}$ \\
\hline
\end{tabular}

Each value is mean \pm standard error of three replicates. Values followed by the same alphabet are not significantly $(p>0.05)$ different from each other using Tukey's Test.

Table 3. Mortality of $C$. maculatus exposed to $0.6 \mathrm{~g}$ dosage of RHA from different rice varieties from Nigeria

\begin{tabular}{cccc}
\hline RHA & \multicolumn{3}{c}{$\%$ adult mortality in hours post treatment } \\
& $\mathbf{2 4}$ & $\mathbf{4 8}$ & $\mathbf{7 2}$ \\
\hline Nupe & $20.00 \pm 0.00^{\mathrm{b}}$ & $43.33 \pm 6.67^{\mathrm{b}}$ & $100.00 \pm 0.00^{\mathrm{c}}$ \\
Jemila & $73.33 \pm 6.67^{\mathrm{e}}$ & $83.33 \pm 3.33^{\mathrm{c}}$ & $100.00 \pm 0.00^{\mathrm{c}}$ \\
FARO 44 & $56.67 \pm 3.33^{\mathrm{d}}$ & $83.33 \pm 6.67^{\mathrm{c}}$ & $100.00 \pm 0.00^{\mathrm{c}}$ \\
Kilaki & $53.33 \pm 3.33^{\mathrm{cd}}$ & $83.33 \pm 3.33^{\mathrm{c}}$ & $100.00 \pm 0.00^{\mathrm{c}}$ \\
Aroso & $40.00 \pm 0.00^{\mathrm{c}}$ & $73.33 \pm 3.33^{\mathrm{c}}$ & $100.00 \pm 0.00^{\mathrm{c}}$ \\
Igbemo & $13.33 \pm 3.33^{\mathrm{ab}}$ & $30.00 \pm 5.77^{\mathrm{b}}$ & $53.33 \pm 3.33^{\mathrm{b}}$ \\
Control & $0.00 \pm 0.00$ & $0.00 \pm 0.00$ & $0.00 \pm 0.00$ \\
\hline
\end{tabular}

Each value is mean \pm standard error of three replicates. Values followed by the same alphabet are not significantly $(\mathrm{p}>0.05)$ different from each other using Tukey's Test.

Table 4. Mortality of C. maculatus exposed to $0.8 \mathrm{~g}$ dosage of RHA from different rice varieties from Nigeria RHA $\%$ adult mortality in hours post treatment

24

\begin{tabular}{cccc} 
& $\mathbf{2 4}$ & $\mathbf{4 8}$ & $\mathbf{7 2}$ \\
\hline Nupe & $30.00 \pm 0.00^{\mathrm{b}}$ & $53.33 \pm 3.33^{\mathrm{c}}$ & $100.00 \pm 0.00^{\mathrm{c}}$ \\
Jemila & $83.33 \pm 3.33^{\mathrm{d}}$ & $100.00 \pm 0.00^{\mathrm{c}}$ & $100.00 \pm 0.00^{\mathrm{c}}$ \\
FARO 44 & $56.67 \pm 3.33^{\mathrm{c}}$ & $100.00 \pm 0.00^{\mathrm{c}}$ & $100.00 \pm 0.00^{\mathrm{c}}$ \\
Kilaki & $46.67 \pm 3.33^{\mathrm{c}}$ & $96.67 \pm 3.33^{\mathrm{d}}$ & $100.00 \pm 0.00^{\mathrm{c}}$ \\
Aroso & $53.33 \pm 3.33^{\mathrm{c}}$ & $86.67 \pm 3.33^{\mathrm{d}}$ & $100.00 \pm 0.00^{\mathrm{c}}$ \\
Igbemo & $20.00 \pm 5.77^{\mathrm{b}}$ & $33.33 \pm 3.33^{\mathrm{b}}$ & $63.33 \pm 3.33^{\mathrm{b}}$ \\
Control & $0.00 \pm 0.00^{\mathrm{a}}$ & $0.00 \pm 0.00^{\mathrm{a}}$ & $0.00 \pm 0.00^{\mathrm{a}}$ \\
\hline
\end{tabular}

48

72 
Evaluation of Rice Husk Ash from Different Rice Varieties from Nigeria: Silica Content and Lethality to Two Storage Beetles

Each value is mean \pm standard error of three replicates. Values followed by the same alphabet are not significantly $(\mathrm{p}>0.05)$ different from each other using Tukey's Test.

Table 5. Mortality of C. maculatus exposed to $1.0 \mathrm{~g}$ dosage of RHA from different rice varieties from Nigeria

\begin{tabular}{cccc}
\hline RHA & \multicolumn{3}{c}{$\%$ adult mortality in hours post treatment } \\
& $\mathbf{2 4}$ & $\mathbf{4 8}$ & $\mathbf{7 2}$ \\
\hline Nupe & $36.67 \pm 3.33^{\mathrm{bc}}$ & $60.00 \pm 0.00^{\mathrm{c}}$ & $100.00 \pm 0.00^{\mathrm{c}}$ \\
Jemila & $86.67 \pm 3.33^{\mathrm{f}}$ & $100.00 \pm 0.00^{\mathrm{c}}$ & $100.00 \pm 0.00^{\mathrm{c}}$ \\
FARO 44 & $76.67 \pm 6.67^{\mathrm{ef}}$ & $100.00 \pm 0.00^{\mathrm{c}}$ & $100.00 \pm 0.00^{\mathrm{c}}$ \\
Kilaki & $60.00 \pm 5.77^{\mathrm{de}}$ & $100.00 \pm 0.00^{\mathrm{c}}$ & $100.00 \pm 0.00^{\mathrm{c}}$ \\
Aroso & $53.33 \pm 3.33^{\mathrm{cd}}$ & $86.67 \pm 3.33^{\mathrm{d}}$ & $100.00 \pm 0.00^{\mathrm{c}}$ \\
Igbemo & $26.67 \pm 3.33^{\mathrm{b}}$ & $40.00 \pm 5.77^{\mathrm{b}}$ & $63.33 \pm 3.33^{\mathrm{b}}$ \\
Control & $0.00 \pm 0.00^{\mathrm{a}}$ & $0.00 \pm 0.00^{\mathrm{a}}$ & $0.00 \pm 0.00^{\mathrm{a}}$ \\
\hline
\end{tabular}

Each value is mean \pm standard error of three replicates. Values followed by the same alphabet

are not significantly $(\mathrm{p}>0.05)$ different from each other using Tukey's Test

the insect within $24 \mathrm{~h}$ followed by FARO 44 and Kilaki

There was variation in the lethal dosage $(\mathrm{g})$ required to achieve $50 \%$ mortality of adult $C$. maculatus by the RHAs from different geographical locations in Nigeria within $24 \mathrm{~h}$ post treatment as presented in Table 6. The Jemila RHA had the lowest $\mathrm{LD}_{50}$ requiring $0.34 \mathrm{~g}$ to achieve $50 \%$ mortality of RHAs with $\mathrm{LD}_{50}$ of $0.50 \mathrm{~g}$ and $0.68 \mathrm{~g}$, respectively. The Chi-square values of the treatments also reflected the level of their effectiveness as they all recorded Chi-square values that was greater than 3.81 .

Table 6. Summary of regression analysis of data upon $24 \mathrm{~h}$ exposure of $C$. maculatus to grain treated with RHA of different rice varieties from Nigeria

\begin{tabular}{ccccccc}
\hline RHA & Slope \pm S.E & Intercept \pm S.E & $\mathrm{X}^{2}$ & LD $_{50}(\mathrm{~g})$ & 95\%FL & Sign \\
\hline Nupe & $1.07 \pm 0.14$ & $-0.57 \pm 0.07$ & 25.33 & 3.39 & $2.34-4.57$ & 0.021 \\
Jemila & $2.17 \pm 0.18$ & $-0.37 \pm 0.07$ & 112.98 & 0.34 & $0.03-0.73$ & 0.0001 \\
FARO 44 & $1.81 \pm 0.18$ & $-0.54 \pm 0.07$ & 95.15 & 0.50 & $0.04-0.93$ & 0.022 \\
Kilaki & $2.33 \pm 0.18$ & $-0.29 \pm 0.07$ & 73.01 & 0.68 & $0.29-0.99$ & 0.0001 \\
Aroso & $0.77 \pm 0.14$ & $-0.36 \pm 0.07$ & 25.23 & 0.75 & $0.32-1.08$ & 0.0001 \\
Igbemo & $0.79 \pm 0.14$ & $-0.86 \pm 0.07$ & 25.43 & 3.46 & $2.81-5.02$ & 0.020 \\
\hline
\end{tabular}

Note: $\mathrm{LD}_{50}=$ lethal dosage; $\mathrm{SE}=$ standard error; $\mathrm{X}^{2}=$ Chi-square; $\mathrm{FL}=$ Fiducial limits.

The mortality of adult $S$. zeamais exposed to different dosages of RHA from different geographical locations in Nigeria is presented in Tables 7-11. The mortality of the insect varied with the type of the RHA, dosage and period of exposure. Table 7 showed that at $0.2 \mathrm{~g}$ dosage, within 24 hours post treatment Jemila, FARO 44, Kilaki and Aroso RHAs, produced $36.67 \%, 33.33 \%, 33.33 \%$ and $30.00 \% \mathrm{~S}$. zeamais mortality, respectively, which was significantly $(\mathrm{p}<$ 0.05 ) different from others. At $72 \mathrm{~h}$ post treatment, all the treatments were able to achieve $50 \%$ or greater beetle mortality. After $96 \mathrm{~h}$ post treatment, Jemila RHA produced the highest mortality of $93.3 \%$ and was significantly different from other treatments. Within $48 \mathrm{~h}$ post treatment, at $0.4 \mathrm{~g}$ dosage Jemila RHA from Kaduna produced $86.6 \%$ mortality of the beetle and was significantly $(\mathrm{p}<0.05)$ different from other RHAs except FARO 44 RHA. At 96 h of exposure, Jemila RHA produced $96.67 \%$ beetle mortality and was significantly different from other RHAs except FARO 44 and Kilaki RHAs which recorded $83.33 \%$ and $76.67 \%$, respectively (Table 8 ). Within $24 \mathrm{~h}$ post treatment at $0.6 \mathrm{~g}$ dosage Jemila RHA achieved up to $50 \%$ mortality of the insect and was significantly $(\mathrm{p}<0.05)$ different from others except Anambra and Bauchi RHAs that recorded $46.67 \%$ mortality. At $96 \mathrm{~h}$ of exposure, Jemila RHA produced $100 \%$ beetle mortality and was significantly different from others except FARO 44 RHA that achieved $93.33 \%$ mortality (Table 9).

Table 10 showed that Jemila RHA was significantly $(\mathrm{p}<0.05)$ different from the other RHAs producing $100 \%$ beetle mortality within $72 \mathrm{~h}$ period of exposure. At $96 \mathrm{~h}$ post treatment, Jemila and FARO 44 RHAs achieved 100\% mortality of $S$. zeamais which was significantly different from others except Kilaki RHA that produced $93.33 \%$ mortality at $0.8 \mathrm{~g}$ dosage (Table 10). Within $72 \mathrm{~h}$ post treatment at $1.0 \mathrm{~g}$ dosage, Jemila RHA achieved $100 \%$ S. zeamais mortality and was significantly $(\mathrm{p}<0.05)$ different from other treatments. At $96 \mathrm{~h}$ post treatment, Jemila, FARO 44 and Kilaki RHAs achieved $100 \%$ insect mortality of the beetle (Table 11). All the treatments recorded above $80 \%$ mortality of the insect within $96 \mathrm{~h}$ post treatment. Regardless of the period of observation, no mortality was observed in controls (Table 7-11).

Table 7. Mortality of $S$. zeamais exposed to $0.2 \mathrm{~g}$ dosage of RHA from different rice varieties from Nigeria

\begin{tabular}{crccc}
\hline RHA & \multicolumn{4}{c}{ \% adult mortality in hours post treatment } \\
& $\mathbf{2 4}$ & $\mathbf{4 8}$ & $\mathbf{7 2}$ & $\mathbf{9 6}$ \\
\hline Nupe & $10.00 \pm 0.00^{\mathrm{a}}$ & $30.00 \pm 0.00^{\mathrm{b}}$ & $56.67 \pm 3.33^{\mathrm{b}}$ & $66.67 \pm 3.33^{\mathrm{bc}}$ \\
Jemila & $36.67 \pm 3.33^{\mathrm{b}}$ & $70.00 \pm 0.00^{\mathrm{d}}$ & $90.00 \pm 5.77^{\mathrm{c}}$ & $93.33 \pm 3.33^{\mathrm{d}}$ \\
FARO 44 & $33.33 \pm 3.33^{\mathrm{b}}$ & $60.00 \pm 0.00^{\mathrm{cd}}$ & $66.67 \pm 3.33^{\mathrm{b}}$ & $76.67 \pm 3.33^{\mathrm{c}}$ \\
Kilaki & $33.33 \pm 3.33^{\mathrm{b}}$ & $53.33 \pm 3.33^{\mathrm{c}}$ & $60.00 \pm 0.00^{\mathrm{b}}$ & $70.00 \pm 0.00^{\mathrm{bc}}$
\end{tabular}




$\begin{array}{lcccc}\text { Aroso } & 30.00 \pm 0.00^{\mathrm{b}} & 50.00 \pm 5.77^{\mathrm{c}} & 60.00 \pm 5.77^{\mathrm{b}} & 66.67 \pm 6.67^{\mathrm{bc}} \\ \text { Igbemo } & 0.00 \pm 0.00^{\mathrm{a}} & 20.00 \pm 0.00^{\mathrm{b}} & 50.00 \pm 5.77^{\mathrm{b}} & 58.00 \pm 0.00^{\mathrm{b}} \\ \text { Control } & 0.00 \pm 0.00^{\mathrm{a}} & 0.00 \pm 0.00^{\mathrm{a}} & 0.00 \pm 0.00^{\mathrm{a}} & 0.00 \pm 0.00^{\mathrm{a}}\end{array}$

Each value is mean \pm standard error of three replicates. Values followed by the same alphabet are not significantly $(p>0.05)$ different from each other using Tukey's Test.

Table 8. Mortality of S. zeamais exposed to $0.4 \mathrm{~g}$ dosage of RHA from different rice varieties from Nigeria

\begin{tabular}{ccccc}
\hline RHA & \multicolumn{4}{c}{ \% adult mortality in hours post treatment } \\
& $\mathbf{2 4}$ & $\mathbf{4 8}$ & $\mathbf{7 2}$ & $\mathbf{9 6}$ \\
\hline Nupe & $16.67 \pm 3.33^{\mathrm{b}}$ & $39.00 \pm 5.77^{\mathrm{c}}$ & $66.67 \pm 3.33^{\mathrm{bc}}$ & $73.33 \pm 3.33^{\mathrm{b}}$ \\
Jemila & $43.33 \pm 3.33^{\mathrm{c}}$ & $86.67 \pm 3.33^{\mathrm{e}}$ & $90.00 \pm 0.00^{\mathrm{d}}$ & $96.67 \pm 3.33^{\mathrm{c}}$ \\
FARO 44 & $40.00 \pm 0.00^{\mathrm{c}}$ & $70.00 \pm 0.00^{\mathrm{de}}$ & $76.67 \pm 3.33^{\mathrm{cd}}$ & $83.33 \pm 3.33^{\mathrm{bc}}$ \\
Kilaki & $36.67 \pm 3.33^{\mathrm{c}}$ & $66.67 \pm 3.33^{\mathrm{d}}$ & $73.33 \pm 3.33^{\mathrm{bc}}$ & $76.67 \pm 3.33^{\mathrm{bc}}$ \\
Aroso & $33.33 \pm 3.33^{\mathrm{bc}}$ & $63.33 \pm 3.33^{\mathrm{cd}}$ & $70.00 \pm 0.00^{\mathrm{bc}}$ & $73.33 \pm 8.82^{\mathrm{b}}$ \\
Igbemo & $10.00 \pm 0.00^{\mathrm{ab}}$ & $26.67 \pm 3.33^{\mathrm{b}}$ & $50.00 \pm 5.77^{\mathrm{b}}$ & $70.00 \pm 0.00^{\mathrm{b}}$ \\
Control & $0.00 \pm 0.00^{\mathrm{a}}$ & $0.00 \pm 0.00^{\mathrm{a}}$ & $0.00 \pm 0.00^{\mathrm{a}}$ & $0.00 \pm 0.00^{\mathrm{a}}$ \\
\hline
\end{tabular}

Each value is mean \pm standard error of three replicates. Values followed by the same alphabet are not significantly $(\mathrm{p}>0.05$ ) different from each other using Tukey's Test.

Table 9. Mortality of $S$. zeamais exposed to $0.6 \mathrm{~g}$ dosage of RHA from different rice varieties from Nigeria

\begin{tabular}{ccccc}
\hline RHA & \multicolumn{4}{c}{ \% adult mortality in hours post treatment } \\
& $\mathbf{2 4}$ & $\mathbf{4 8}$ & $\mathbf{7 2}$ & $\mathbf{9 6}$ \\
\hline Nupe & $20.00 \pm 0.00^{\mathrm{b}}$ & $43.33 \pm 6.67^{\mathrm{b}}$ & $73.33 \pm 3.33^{\mathrm{bc}}$ & $80.00 \pm 0.00^{\mathrm{bc}}$ \\
Jemila & $50.33 \pm 3.33^{\mathrm{d}}$ & $70.00 \pm 5.77^{\mathrm{c}}$ & $96.67 \pm 3.33^{\mathrm{d}}$ & $100.00 \pm 0.00^{\mathrm{d}}$ \\
FARO 44 & $46.67 \pm 3.33^{\mathrm{cd}}$ & $70.00 \pm 0.00^{\mathrm{c}}$ & $83.33 \pm 3.33^{\mathrm{cd}}$ & $93.33 \pm 3.33^{\mathrm{cd}}$ \\
Kilaki & $46.67 \pm 3.33^{\mathrm{cd}}$ & $66.67 \pm 3.33^{\mathrm{c}}$ & $80.00 \pm 5.77^{\mathrm{c}}$ & $83.33 \pm 3.33^{\mathrm{bc}}$ \\
Aroso & $40.00 \pm 0.00^{\mathrm{c}}$ & $56.67 \pm 3.33^{\mathrm{bc}}$ & $73.33 \pm 3.33^{\mathrm{bc}}$ & $83.33 \pm 6.67^{\mathrm{bc}}$ \\
Igbemo & $13.33 \pm 3.33^{\mathrm{ab}}$ & $30.00 \pm 5.77^{\mathrm{b}}$ & $53.33 \pm 3.33^{\mathrm{b}}$ & $73.33 \pm 3.33^{\mathrm{b}}$ \\
Control & $0.00 \pm 0.00^{\mathrm{a}}$ & $0.00 \pm 0.00^{\mathrm{a}}$ & $0.00 \pm 0.00^{\mathrm{a}}$ & $0.00 \pm 0.00^{\mathrm{a}}$ \\
\hline
\end{tabular}

Each value is mean \pm standard error of three replicates. Values followed by the same alphabet are not significantly $(p>0.05)$ different from each other using Tukey's Test.

Table 10. Mortality of $S$. zeamais exposed to $0.8 \mathrm{~g}$ dosage of RHA from different rice varieties from Nigeria

\begin{tabular}{ccccc}
\hline RHA & \multicolumn{4}{c}{ \% adult mortality in hours post treatment } \\
& $\mathbf{2 4}$ & $\mathbf{4 8}$ & $\mathbf{7 2}$ & $\mathbf{9 6}$ \\
\hline Nupe & $30.00 \pm 0.00^{\mathrm{b}}$ & $53.33 \pm 3.33^{\mathrm{c}}$ & $76.67 \pm 3.33^{\mathrm{bc}}$ & $83.33 \pm 3.33^{\mathrm{b}}$ \\
Jemila & $53.00 \pm 5.77^{\mathrm{c}}$ & $73.33 \pm 6.67^{\mathrm{d}}$ & $100.00 \pm 0.00^{\mathrm{c}}$ & $100.00 \pm 0.00^{\mathrm{c}}$ \\
FARO 44 & $46.67 \pm 3.33^{\mathrm{c}}$ & $73.33 \pm 3.33^{\mathrm{d}}$ & $86.67 \pm 3.33^{\mathrm{c}}$ & $100.00 \pm 0.00^{\mathrm{c}}$ \\
Kilaki & $46.67 \pm 3.33^{\mathrm{c}}$ & $70.00 \pm 5.77^{\mathrm{d}}$ & $83.33 \pm 3.33^{\mathrm{c}}$ & $93.33 \pm 3.33^{\mathrm{bc}}$ \\
Aroso & $43.33 \pm 3.33^{\mathrm{bc}}$ & $70.00 \pm 5.77^{\mathrm{d}}$ & $80.00 \pm 0.00^{\mathrm{c}}$ & $86.67 \pm 3.33^{\mathrm{b}}$ \\
Igbemo & $20.00 \pm 5.77^{\mathrm{b}}$ & $33.33 \pm 3.33^{\mathrm{b}}$ & $63.33 \pm 3.33^{\mathrm{b}}$ & $83.33 \pm 3.33^{\mathrm{b}}$ \\
Control & $0.00 \pm 0.00^{\mathrm{a}}$ & $0.00 \pm 0.00^{\mathrm{a}}$ & $0.00 \pm 0.00^{\mathrm{a}}$ & $0.00 \pm 0.00^{\mathrm{a}}$ \\
\hline
\end{tabular}

Each value is mean \pm standard error of three replicates. Values followed by the same alphabet are not significantly ( $\mathrm{p}>0.05$ ) different from each other using Tukey's Test.

Table 11. Mortality of $S$. zeamais exposed to $1.0 \mathrm{~g}$ dosage of RHA of different rice varieties from Nigeria

\begin{tabular}{cccccc}
\hline RHA & \multicolumn{4}{c}{ \% adult mortality in hours post treatment } \\
& $\mathbf{2 4}$ & $\mathbf{4 8}$ & $\mathbf{7 2}$ & $\mathbf{9 6}$ \\
Nupe & $36.67 \pm 3.33^{\mathrm{bc}}$ & & $60.00 \pm 0.00^{\mathrm{c}}$ & $83.33 \pm 3.33^{\mathrm{b}}$ & $93.33 \pm 6.67^{\mathrm{b}}$ \\
Jemila & $83.67 \pm 3.33^{\mathrm{f}}$ & & $93.33 \pm 3.33^{\mathrm{d}}$ & $100.00 \pm 0.00^{\mathrm{c}}$ & $100.00 \pm 0.00^{\mathrm{b}}$ \\
FARO 44 & $66.67 \pm 3.33^{\mathrm{de}}$ & & $86.67 \pm 3.33^{\mathrm{d}}$ & $90.00 \pm 5.77^{\mathrm{bc}}$ & $100.00 \pm 0.00^{\mathrm{b}}$ \\
Kilaki & $60.00 \pm 5.77^{\mathrm{de}}$ & & $83.33 \pm 3.33^{\mathrm{cd}}$ & $90.00 \pm 0.00^{\mathrm{bc}}$ & $100.00 \pm 0.00^{\mathrm{b}}$ \\
Aroso & $53.33 \pm 3.33^{\mathrm{cd}}$ & & $80.00 \pm 5.77^{\mathrm{cd}}$ & $86.67 \pm 3.33^{\mathrm{bc}}$ & $96.67 \pm 3.33^{\mathrm{b}}$ \\
Igbemo & $26.67 \pm 3.33^{\mathrm{b}}$ & & $40.00 \pm 5.77^{\mathrm{b}}$ & $76.67 \pm 3.33^{\mathrm{b}}$ & $86.67 \pm 3.33^{\mathrm{b}}$ \\
Control & $0.00 \pm 0.00^{\mathrm{a}}$ & & $0.00 \pm 0.00^{\mathrm{a}}$ & $0.00 \pm 0.00^{\mathrm{a}}$ & $0.00 \pm 0.00^{\mathrm{a}}$ \\
\hline
\end{tabular}

Each value is mean \pm standard error of three replicates. Values followed by the same alphabet are not significantly ( $\mathrm{p}>0.05$ ) different from each other using Tukey's Test.

Summary of regression analysis of data upon $24 \mathrm{~h}$ exposure of $S$. zeamais to grain treated with RHA of different rice varieties from Nigeria is presented in Table 12. Low dosage of each ash was required to achieve high mortality of the insect. Jemila RHA was most effective as the lowest amount 
of $0.55 \mathrm{~g}$ was required to achieve $50 \%$ mortality of the insect within $24 \mathrm{~h}$ while Igbemo RHA from Ekiti was least effective requiring $5.16 \mathrm{~g}$ to achieve $50 \%$ insect mortality and was highly significantly different $(\mathrm{p}<0.0001)$ compared to some other treatments. The Chi-square values of the treatments also reflected the level of their effectiveness as all of them recorded Chi square values that were greater than 3.81 . The slope and intercept of the treatments showed that the treatments are very effective as their values are very low.

Table 12: Summary of regression analysis of data obtained upon $24 \mathrm{~h}$ exposure of $\mathrm{S}$. zeamais to grain treated with RHA of different rice varieties from Nigeria

\begin{tabular}{ccccccc}
\hline RHA & Slope \pm S.E & Intercept \pm S.E & $\mathbf{X}^{\mathbf{2}}$ & $\mathbf{L D}_{\mathbf{5 0}}$ & $\mathbf{9 5 \%} \mathbf{F L}$ & Sign \\
\hline Nupe & $1.09 \pm 0.14$ & $0.04 \pm 0.06$ & 34.00 & 4.92 & $3.36-5.36$ & 0.000 \\
Jemila & $0.12 \pm 0.15$ & $0.64 \pm 0.07$ & 37.03 & 0.55 & $0.04-0.93$ & 0.0001 \\
FARO 44 & $1.85 \pm 0.14$ & $0.22 \pm 0.07$ & 45.83 & 0.67 & $0.30-1.07$ & 0.0001 \\
Kilaki & $1.10 \pm 0.14$ & $0.02 \pm 0.06$ & 28.67 & 0.85 & $0.40-1.13$ & 0.007 \\
Aroso & $1.98 \pm 0.13$ & $-0.26 \pm 0.06$ & 25.34 & 0.95 & $0.54-2.30$ & 0.021 \\
Igbemo & $2.05 \pm 0.14$ & $-0.52 \pm 0.07$ & 9.56 & 5.16 & $4.74-6.73$ & 0.729 \\
\hline
\end{tabular}

Note: $\mathrm{LD}_{50}=$ lethal dosage; $\mathrm{SE}=$ standard error; $\mathrm{X}^{2}=$ Chi-square; $\mathrm{FL}=$ Fiducial limits.

Computed correlation coefficients ( $\mathrm{r}$ ) indicating the relationship between silica content and beetle mortality $24 \mathrm{~h}$ post treatment at various dosages of the different RHAs are presented in Table 13. Irrespective of beetle species and dosage, mortality produced was significantly $(\mathrm{p}<0.001)$ positively correlated with silica content of the RHAs.

Table 13. Correlation coefficients showing relationships between silica content and beetle mortality $24 \mathrm{~h}$ post treatment at various dosages of RHAs from Nigeria

\begin{tabular}{ccc}
\hline Dosage (g/20 g of grain) & \multicolumn{2}{c}{ Value of Correlation Coefficients** } \\
& C. maculatus & S. zeamais \\
\hline $\mathbf{0 . 2}$ & 0.943 & 0.952 \\
$\mathbf{0 . 4}$ & 0.933 & 0.961 \\
$\mathbf{0 . 6}$ & 0.955 & 0.959 \\
$\mathbf{0 . 8}$ & 0.865 & 0.973 \\
$\mathbf{1 . 0}$ & 0.941 & 0.948 \\
\hline
\end{tabular}

$* * \mathrm{p}<0.001$

\section{DISCUSSION}

The results obtained in this study indicate significant differences in the silica content of RHA from different varieties of rice grown in different geographical areas of Nigeria. The percentage silica content ranged from about $50 \%$ to $90.45 \%$ and was highest in Jemila rice from Kaduna State, and lowest in Igbemo rice variety from Ekiti State. The results are consistent with the findings of [15] in which geographical location and type of fertilizer used for cultivation of rice were found to have effect on the silica content of RHA. Differences in silica content of RHA obtained from some other rice varieties in Nigeria have similarly been reported by some workers in Nigeria [16] and some other countries such as Sri Lanka [17]. The significant variation in the silica content amongst the rice varieties is presumably due to differences in the soil chemistry of the different geographical locations as suggested by [18] as well as inherent genotypic differences in silicon uptake from the soil, as observed by [19]. Soil chemical characteristics of the different geographical locations where these rice varieties were grown was not determined in this study; neither were their genotypic capabilities in nutrient uptake examined. However, intensive rice crop production can deplete soil available silicon and differences in the intensity of rice cultivation in the various agro-ecological zones of Nigeria have been reported [20]. This may therefore partly explain the differences in the silica content of the RHA of the rice varieties in this study.

Observations on the mortality of adults of C. maculatus and $S$. Zeamais exposed to the different doses of each of the RHA of the rice varieties from six different geographical locations in Nigeria revealed that they were all lethal to the beetles. This has further substantiated earlier reports by some workers that RHA can cause mortality of adult storage beetles exposed to it [21] [(2] (11) [22]. Generally, the higher the dosage, the higher was the mortality inflicted. Of the six different RHAs, that of the rice variety obtained from Kaduna (Jemila rice variety) generally inflicted higher mortality than others, 24 hours post exposure. This superiority was clearly reflected in its lowest amount required to achieve $50 \%$ mortality $\left(\mathrm{LD}_{50}\right)$ of the beetles. Ashes obtained from many other plant materials have also been reported as lethal to adult storage beetles. This includes wood ash [3] [4], ash from Acacia sp. and Bombyx sp. [23], leaf ashes of Cupressus arizonica, Eucalyptus grandis, Ocimum gratissimum, and root ash of Vetivera zizanoides [6], ash of Newbouldia laevis and Bablash [24], ash from Lophira lanceolata and 
Hymenocardia acida [11] and wood ash from Hymenocardia acida [8].

The lethal action of RHA on adult beetles has been explained by various speculations, conjectures and adult behavioural manifestations. [25] suggested that inert substances generally cause a loss of body moisture. [2] reported that adults of $C$. maculatus exposed to RHA manifested significant weight loss (more than half of the body weight was lost within 2 days) and died. RHA has also been reported to contain numerous needle-like particles presumably derived from the setae covering the outer surface of the rice husk [10]. These needle-like particles may trigger a physical reaction on the integument of the insects and the resulting physical disturbance may help cause their death [2]. [2] further reported a manifest display of dislike and aversion seen amongst beetles exposed to RHA, involving extension of the membranous hind wings presumably in preparation for quick escape from captivity in the arena. Adult beetles exposed to RHA may also could have been weakened or even killed due to abrasion of the integument and consequent dehydration and desiccation [26] [2]. [11] also agreed that, during insects crawling over the treated grains, the chemical constituents of ash could lodge between cuticular segments and increase water loss through abrasion of the cuticle. The high silica $\left(\mathrm{SiO}_{2}\right)$ of RHA is further believed to have a lethal effect on insects including irritation and desiccation of adult insects [27] [1]. Therefore, it can be presumed that the higher silica content of Jemila rice husk ash (from Kaduna State) may partly explain its higher lethality to the adult beetles in comparison with other RHAs, observed in this study. Correspondingly, irrespective of beetle species and dosage, adult mortality was computed in this study to be significantly positively correlated with silica content of the RHAs. Diatomaceous earth (DE) is known to contain silica in amounts similar to highly siliceous RHA $\left(\mathrm{SiO}_{2}>90 \%\right)$ [10]. The primary mechanism for the lethal action of DE against adult beetles is effective adsorption of lipids and fatty acids of the epicuticle of the insect integument, which leads to death [12]. The same may also be operative with RHA in this study, but however, requires empirical verification.

C. maculatus was generally more susceptible to the RHAs than $S$. zeamais. This is clearly indicated by computed $\mathrm{LD}_{50}$. For each RHA, the quantity required to achieve 50\% mortality of $S$. zeamais was more than the quantity required for $C$. maculatus. For instance, with JRHA the $\mathrm{LD}_{50}$ for $C$. maculatus was $0.34 \mathrm{~g}$ whereas it was $0.55 \mathrm{~g}$ for $S$. zeamais. This observation is consistent with the report of [28] that $S$. granarius and $S$. zeamais were more tolerant to exposure to RHA and DE than Lasioderma serricorne and C. maculatus. It has also been reported that the lesser grain borer Rhyzopertha dominica (F.) was more tolerant to rice husk ash toxicity than $S$. zeamais [22]. It has been suggested that taxonomic differences in the nature and characteristics of the integument of adult storage beetles causing differential rates of water loss, may partly explain their differential susceptibility to insecticidal pulverized plant materials [29]. It has been suggested that a cocktail of insecticidal plant materials may be more formidable in mitigating insect pest problems in stored grain [30]. Unequivocally, RHA, especially those with high silica content such as Jemila RHA identified in this study, may be good candidates for inclusion in such insecticidal cocktails.

\section{REFERENCES}

[1] Ajayi, K., Kalyani, M., Devendra, K. and OM, P. 2012. Properties and industrial applications of rice husk: A review. International Journal of Emerging Technology and Advanced Engineering 2(10): 86-90.

[2] Ofuya, T.I. and Adler, C.S. 2014. Ability of rice husk and husk ash powders to protect cowpea seeds against Callosorbruchus maculatus Fabricius (Coleoptera: Chrysomelidae: Bruchinae) damage. Journal of Sustainable Technology 5, 70-79

[3] Ofuya, T.I. 1986. Use of wood ash, dry chilli pepper fruits and onion scale leaves in reducing Callosobruchus maculatus (Fabricius) damage in cowpea seeds during storage. Journal of Agricultural Science, Cambridge 107: 467-468.

[4] Wolfson, J.L., Shade, R.E., Mentzer, P.E. and Murdock, L.L. 1991. Efficacy of ash for controlling infestations of Callosobruchus maculatus (F.) (Coleoptera: Bruchidae) in stored cowpeas. Journal of Stored Products Research 27: 239-243.

[5] Apuuli, J.K.K. and Villet, M.H. (1996). The use of wood ash for the protection of stored cowpea seed (Vigna unguiculata (L.) Walp) against Bruchidae (Coleoptera). African Entomology4: 97-98.

[6] Akob, C.A. and Ewete, F.K. 2007. The efficacy of ashes four locally used plant materials against Sitophilus zeamais (Coleoptera: Curculionidae) in Cameroon. International Journal of Tropical Insect Science 27: 21-26.

[7] Baoua, I.B., Amadou, L., Margam, V. and Murduck, L.L. 2012. Comparative evaluation of six storage methods for postharvest preservation of cowpea grain. Journal of Stored Products Research 49: $171-175$

[8] Goudoungou, J.W., Nukenine, E.N., Suh, C., Gangué, T., and Ndjonka, D. 2018. Effectiveness of binary combinations of Plectranthus glandulosusleaf powder and Hymenocardia acida wood ash against Sitophilus zeamais (Coleoptera: Curculionidae). Agriculture \& Food Security 7: 26. https://doi.org/10.1186/s40066-018-0179-z

[9] Tee, S.P. (1981). Powdered paddy husk ash for grain protection against stored product beetles. MAPPS Newsletter 5: 2-3.

[10] Naito, A. (1999). Developing cost-free technology for controlling soybean insect pests in Indonesia. FFTC Extension Bulletin 468.

[11] Mazarin, A., Nukenine, E.N., Niu, C., and Vincent, F.V. 2016. Synergistic Effects of Wood Ash and Essential Oil on Fecundity, Pupal Eclosion and Adult Mortality of Callosobruchus maculatus (Coleoptera: Bruchidae), Cowpea Seed Weevil. American Journal of Experimental Agriculture 11(6): 1-12, 2016; Article no.AJEA. 25306

[12] Shah, M.A. and Khan, A.A. 2014. Use of diatomaceous earth for the management of stored

[13] Monti, A., DI Virgilio, N. and Venturi, G. 2008. Mineral composition and ash content of six major energy crops. Biomass and Energy 32: 216-223.

[14] Finney, D.J. (1971). Probit Analysis. Cambridge University Press, Cambridge, London, 333pp.

[15] Bining, A. S. and Jenkins B. M. (1992): Thermo chemical reaction kinetics for rice straw from an approximate integral technique. American Society of Agricultural Engineers. Meeting (USA). No. 92-6001/92-6040

[16] Abiodun, Y.O. and Jimoh, A.A. 2018. Microstructural characteristics, physical and chemical properties of rice husk ash as viable pozzolan in building materials: A case study of some Nigerian grown rice varieties. Nigerian Journal of Technology 37 (1): 71-77.

[17] Virajini, R.M.S., Perera, V.P.S. and Rajendra, J.C.N. 2014. Physical properties and content of silica in different varieties of rice husk found in Sri Lanka. Proceedings of the Technical Session 30 (2014): 105-110, Institute of Physics, Sri Lanka.

[18] Muntohar A. S. 2002: Utilization of uncontrolled burnt rice husk ash in soil improvement. Dimensi Teknik Sipil. 4(2): 100-105.

[19] [19] Ma, J.F., Yamaji, N., Tamai, K and Mitanir, N. 2007. Genotypic difference in silicon uptake and expression of silicon transporter genes in rice. Plant Physiology 145 (3): 919-924.

[20] Abe, S.S., Yamasaki, Y. and Wakatsuki, T. 2016. Assessing silicon availability in soils of rice-growing lowlands and neighbouring uplands in Benin and Nigeria. Rice Science 23 (4): 196-202.

[21] Adebayo, T.A. and Ibikunle, O. 2014. Potential of rice husk ash, cowdung ash and powdered clay as grain protectants against Callosobruchus maculatus (F.) and Sitophilus zeamais (Mots). Applied Tropical Agriculture 19 (2): 48-53. 
[22] Otitodun, G.O., Opit, G.P., Nwaubani, S.I. and Okonkwo, E.U. 2017. Efficacy of rice husk ash against rice weevil and lesser grain borer on stored wheat. African Crop Science Journal 25 (2): 145-155.

[23] Ekesi 2005. Ash from Cooking fire Synergizes Efficacy of the Entomopathogenic fungus, Metarhizium anisopliae against Four or Stored Product Insect Pests. International Centre of Insect Physiology and Ecology 22: $64-75$.

[24] Ogungbite O. C., Odeyemi O. O. and Ashamo M. O. (2014): Powders of Newbouldia laevis as protectants of cowpea seeds against infestation by Callosobruchus maculatus (Fab.) for poor

[25] Subramanyam, B.H. and Roesli, R. 2000. Inert dusts. In Subramanyam, B.H., and Hagstrum, D.W. (Editors), Alternatives to pesticides in stored-product IPM. Dordrecht (The Netherlands): Kluwer Academic Publishers, pp. 321-380.

[26] Tadesse A. and Basedow Th. (2005): Laboratory and field studies on the effect of natural control measures against insect pests in stored maize in Ethiopian Journal of Plant Diseases and Protection 112(2):156-172.

[27] Kalapathy, U., Proctor, A. and Shultz, J. 2000. A simple method for production of pure silica from rice hull ash. BioresourceTechnology 73 : 257-262.

[28] Ofuya, T. and Adler, C. 2018. Comparative lethality of rice husk ash and a diatomaceous earth to adults off our storage beetles. Julius-Kuehn Archiv 463: 823-829.

[29] Ogungbite O. C. and Oyeniyi E. A. 2014. Newbouldia laevis(Seem) as an entomocide against Sitophilus oryzae and Sitophilus zeamais infesting maize grain. Jordan Journal of Biological Science, 7 (1): 49-55.

[30] Ofuya, T.I. 2019.The potential for integration of insecticidal botanical products with other control methods for stored grain protection against insect infestation and damage in Nigeria. IOBC Conference, Pisa, Italy. 